DOI 10. $18307 / 2016.0610$

(c) 2016 by Journal of Lake Sciences

\title{
三峡澎溪河高阳平湖高水位期间磷-藻生态模型研究”
}

\author{
赫 斌 ${ }^{2,3}$, 李 哲 ${ }^{1,2,4}$, 冯 婧 2,3 , 肖 艳 ${ }^{1,2 * *}$, 郭劲松 1,2 \\ (1: 中国科学院重庆绿色智能技术研究院,重庆 400714) \\ (2: 中国科学院水库水环境重点实验室,重庆 400714) \\ (3: 重庆大学城市建设与环境工程学院,重庆 400045) \\ (4:中国长江三峡集团公司,北京 100038)
}

摘 要: 通过在前期建立的总磷模型基础上建立磷-藻模型. 从正磷酸盐所代表的无机磷和藻类生物量所代表的有机磷 人手, 分析蓄水期所累积的磷在高水位时期各种形态转化和循环机制, 以期从定性和定量两方面说明冬季末水华暴发所 消耗磷的来源. 研究表明: 高水位蓄水期、稳定期和水华期这 3 个阶段中各磷库磷通量各不相同. 淹没消落带磷释放是该 时期高阳平湖溶解性正磷酸盐的主要来源 (占总输人通量的 $72.69 \%$ ), 其释放量与被淹没消落带面积和消落带水土界面 磷交换速率有关, 淹没消落带面积越多, 磷交换速率越高, 磷释放量越大, 对整个磷循环的贡献越大. 但水华期间藻磷的 合成率最大仅为 $14.4 \%$, 藻类对冬季高水位期间所积累磷的利用量依然相对较低.

关键词: 三峡库区;澎溪河;高阳平湖;磷-藻模型;磷循环;形态转化

\section{Study on the phosphorus-algal ecological modelling during high water level period in Lake Gaoyang of Pengxi River, Three Gorges Reservoir}

\author{
HE Bin $^{2,3}$, LI Zhe ${ }^{1,2,4}$, FENG Jing ${ }^{2,3}$, XIAO Yan ${ }^{1,2 * *}$ \& GUO Jinsong ${ }^{1,2}$ \\ ( 1: Chongqing Institute of Green and Intelligent Technology, Chinese Academy of Sciences, Chongqing 400714, P.R.China) \\ (2: Key Laboratory of Water Environment Reservoir, Chinese Academy of Sciences, Chongqing 400714, P.R.China) \\ (3: Faculty of Urban Construction and Environmental Engineering, Chongqing University, Chongqing 400045, P.R.China) \\ (4: China Three Gorges Corporation, Beijing 100038, P.R. China)
}

\begin{abstract}
The article set up model of phosphorus-algae on the basis of establishing total phosphorus model in early period. From the start of inorganic salts represented by orthophosphate and organophosphorus represented by algal biomass, it analyses at high water level stages, kinds of form transformation and cycle mechanism of accumulated phosphorus due to impounding stage in order to explain the source of the phosphorus which is consumed by the outbreak of water bloom at the end of winter from fixed quantity and fixed nature two parts. The research shows that the total phosphorus of phosphorus pool in the three phases of impounding stage, stable stage and algae bloom stages at high water level in Lake Gaoyang is totally different. Water-level-fluctuating zone with phosphorus release is a major source of solubility reactive phosphorus at high water level in Lake Gaoyang ( $72.69 \%$ of the total input flux), which is related to the area of water-level-fluctuating zone and the phosphorus exchange rate in the soil and water interface of water-level-fluctuating zone. More flooded area, the higher exchange rate of phosphorus, more phosphorus release, the greater contribution of the phosphorus cycle. And the maximum synthetic ratio of phosphorus-algae was only $14.4 \%$. However, the amount of utilized phosphorus at algal bloom stage was small.
\end{abstract}

Keywords: Three Gorges Reservoir; Pengxi River; Lake Gaoyang; phosphorus-algal model; phosphorus cycle; morphological transformation

* 国家自然科学基金项目(51309220,51679226)和重庆市基础科学和前沿技术研究专项 ( cstc2015jcyjBX0006)联合 资助. 2016-02-25 收稿;2016-02-27 收修改稿.赫斌( 1991 ) ,男,硕士研究生;E-mail:binhe85@ 163.com.

** 通信作者;E-mail: yxiao@ cigit.ac.cn. 
近年来,三峡支流回水区频繁出现的水华现象备受关注. 本质上,水华过程是藻类在过量养分供给与充 裕能量输人基础上大量增殖而成. 尽管近年来的主流观点普遍认为, 在营养盐浓度已经充足的条件下, 三峡 支流回水区水华过程主要受到光、热输人和水动力条件的驱动而形成 ${ }^{[1-3]}$. 但营养盐与藻类生长之间的定量 关系却未有更深层次的分析探讨.

三峡水库 “蓄清排浑” 的调度运行方案将在水库库周形成垂直落差达 $30 \mathrm{~m}$ 、总面积约为 $400 \mathrm{~km}^{2}$ 的水库 消落带. 消落带在水库运行下积极参与水体中营养盐的分布与循环过程, 成为三峡水库水体营养盐源汇的 重要场所 ${ }^{[4-6]}$. 前期分析发现, 三峡水库汛末蓄水后水体中总磷 ( TP ) 易随着水位升高与回水顶托出现显著 的积累现象 ${ }^{[7-8]}$. 方芳等研究表明, 尽管水体中 TP 浓度呈现略微增加或并不呈现显著增加, 但因总库容的显 著增加, 回水区中 TP 总量显著提高 ${ }^{[9]}$. 另一方面, 随冬季末期气温回暖, 通常在初春第一场明显降雨导致的 “倒春寒”气候现象来临之前, 三峡库区支流库湾易出现 1 次显著水华过程,近年来发现的主要优势藻为硅 藻. 由于水华过程、藻类增殖将显著改变水体中磷形态, 并影响磷通量. 故在前期完成澎溪河高阳平湖冬季 $\mathrm{TP}$ 收支模型和冬季藻-磷关系的碱性磷酸酶分析基础上 ${ }^{[10]}$, 本研究构建澎溪河冬季高水位时期的藻-磷模 型, 分析冬季水华过程磷通量关系, 揭示冬季末期水华过程磷形态与转化特征. 研究将为进一步阐释三峡支 流高水位时期磷的生物地球化学过程提供科学基础.

\section{1 材料与方法}

\section{1 研究区域与样品采集}

澎溪河地处四川盆地东部边缘, 流域面积 $5173 \mathrm{~km}^{2}$, 干流全长 $182.4 \mathrm{~km}$. 本研究选择高阳平湖入口 ( $31^{\circ}$ $6^{\prime} 12.01^{\prime \prime} \mathrm{N}, 108^{\circ} 39^{\prime} 18.18^{\prime \prime} \mathrm{E}$, 简写为 “高阳 $1^{\prime}$ ”) 、湖心 ( $31^{\circ} 5^{\prime} 48.2^{\prime \prime} \mathrm{N}, 108^{\circ} 40^{\prime} 20.1^{\prime \prime} \mathrm{E}$, 简写为 “高阳 2”) 和出口 ( $31^{\circ} 4^{\prime} 59.77^{\prime \prime} \mathrm{N}, 108^{\circ} 40^{\prime} 13.58^{\prime \prime} \mathrm{E}$, 简写为“高阳 3”) 为控制性断面 (图 2). 分别以 $1 、 3$ 号点位为进、出水点, 重 点进行对 2 号点位的磷-藻动态模拟. 于 2011 年 9 月- 2012 年 3 月一个完整的高水位运行阶段开展跟踪观 测. 采样频次为 1 次/周,采样时间控制在当天 $9: 30-16: 30$.

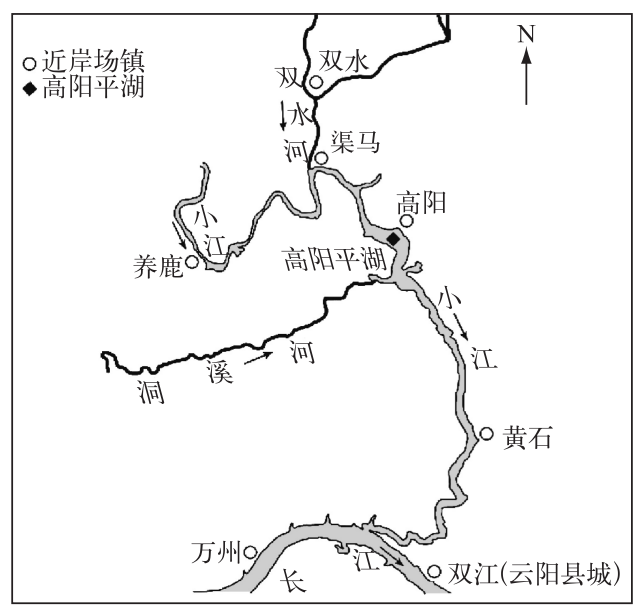

图 1 澎溪河回水区云阳段及高阳平湖示意

Fig.1 Backwater area of the Pengxi

River and Lake Gaoyang

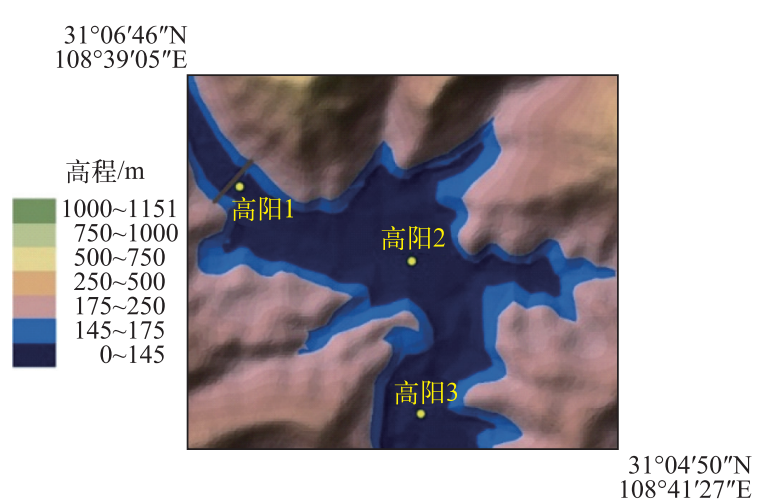

图 2 高阳平湖水质指标采样点位置

Fig. 2 Distribution of sampling sites for water-quality indicators in Lake Gaoyang

消落带土壤磷释放的研究是在前期工作的基础上进行的 ${ }^{[11-12]}$, 采集地点在高阳平湖库湾平台附近高程 分别为 $150 、 155 、 160 、 165 \mathrm{~m}$ 处的消落带斜坡面分梯度采样. 采样使用柱状采样器, 采样管高 $55 \mathrm{~cm}$, 内径 $10.5 \mathrm{~cm}$, 然后切取 $0 \sim 20 \mathrm{~cm}$ 的表层样品放于自封袋中冷藏保存带回实验室, 用于分析消落带土壤的活性磷; 在高阳平湖库湾平台附近水面靠近河床处使用柱状采样器采集底泥, 采集后将样品放于自封袋中冷藏保存 带回实验室用于测定底泥的活性磷. 土壤监测时间为 2011 年 9 月至 2012 年 3 月一个完整的高水位时期,采 
样频次为 1 次/月,采样时间控制在当天 $9: 30-16: 30$.

根据前期研究分析 ${ }^{[10]}$, 将高水位时期 ( 9 月中旬至次年 3 月) 划分为 3 个阶段: 蓄水期 ( 9 月中旬一 10 月 下旬）、稳定期 ( 11 月上旬 -1 月下旬) 和水华期 ( 2 月上旬 -3 月下旬).

\section{2 测试指标与分析方法}

测定指标包括: 水温、水面人射光照强度、溶解性正磷酸盐 $\left(\mathrm{PO}_{4}^{3-}-\mathrm{P}\right)$ 、叶绿素 a ( Chl.a )、消落带活性磷 $\left(p s_{1}\right)$ 、永久淹没区活性磷 $\left(p s_{2}\right)$ 等. $p s_{1}$ 和 $p s_{2}$ 的计算方法为: 总磷 - 无机磷 + 颗粒物表面的吸附磷 + 铝磷+铁 磷 ${ }^{[13]}$; 土壤总磷和无机磷采用磷锄蓝比色法 ${ }^{[12]}$ 进行测定; 吸附磷、铝磷、铁磷采用 SMT 连续浸提法 ${ }^{[14]}$ 进行 测定; 所有水质化学测试指标分析方法参照《水和废水监测分析方法 (第四版) 》进行测定 ${ }^{[13]}$, 所有土壤化学 测试指标分析方法参照《土壤农业化学分析方法》进行测定 ${ }^{[14]}$. 另外, 叶绿素 a 浓度 (Chl.a) 为 $500 \mathrm{ml}$ 原水 经 GF/C 膜过滤后用 $90 \%$ 丙酮萃取后测定.

参考李哲 ${ }^{[15]}$ 在澎溪河回水区建立的藻类生物量湿重 $(B i o M)$ 预测模型, 通过测定 Chl.a 浓度换算为藻类 生物量湿重, 藻类生物量湿重 $(B i o M)$ 的计算公式为:

$$
\lg (\text { BioM })=0.7563 \cdot \lg (\text { Chl.a })+2.7331\left(R^{2}=0.7704, n=212, \text { Sig. } \leqslant 0.01\right)
$$

一般认为, 藻类生物量湿重中含磷量为 $0.00628 \mathrm{~g} / \mathrm{g}^{[16-17]}$, 则藻磷的计算公式为:

$$
\text { 藻磷 }=0.00628 \cdot \text { BioM }
$$

式中, Chl. $a$ 和 BioM 单位为 $\mathrm{g} / \mathrm{m}^{3}$; 藻磷单位为 $\mathrm{g} / \mathrm{m}^{3} ; n$ 为样本数; Sig. 为显著性水平.

\section{3 数据来源与分析方法}

本文所有数据均录人 Excel 和 Origin 软件进行统计分析, 模型部分使用 MATLAB 7.0 进行数据处理, 采 用变异系数 $C V$ 值表征数据变化幅度. 模型计算中所需的水位数据来自于中国长江三峡集团网站; 日流量数 据根据开县温泉水文站 (占 $24 \%$ 控制流域面积) 流量实测数据进行换算; 气候气象数据直接来自于研究团队 设置在高阳平湖库湾的气象站. 不同水位下的高阳平湖库容、水域面积和消落带实际面积提取, 以 $175 \mathrm{~m}$ 以 下 $1: 2000 D E M$ 为基础,采用 ArcGIG 直接提取.

\section{2 高水位期间高阳平湖磷-藻生态模型的构建}

\section{1 时空边界与模型基本假设}

本研究集中于揭示冬季蓄水后高水位时期的藻磷循环收支关系, 故研究的时间边界为 9 月至次年 3 月 整个高水位运行期间, 空间边界为高阳平湖高阳 1 、高阳 3 控制断面内的 $175 \mathrm{~m}$ 高程以下的水域、永久淹没 区与消落带. 模型具有以下假设:

1) 因研究期间为冬季, 高阳平湖水体不分层且枯水期未出现异重流等改变水质空间分布的现象 ${ }^{[18]}$, 故 水域内不存在水平和垂直空间位置差异, 假设变量与参数均为空间均质性 ${ }^{[8,18-19]}$. 不考虑因水动力学条件改 变而产生的各种生态要素时空迁移特征, 即所构建的磷-藻模型为零维 ${ }^{[9,19]}$.

2) 因研究期间为冬季枯水季节, 期间降水量仅为全年的 10\% 20\%, 且相比较于高阳平湖水域面积, 高 阳 1 高阳 3 断面间控制流域面积很小; 云阳县高阳污水处理厂排污口位于高阳 3 断面下游 $400 \mathrm{~m}$ 处, 研究 区域内没有化工厂, 故模型中忽略面源、点源等外源性污染导致的磷输人.

3) 在模型设计中以 $1 \mathrm{~d}$ 为一个稳态单位, 将高水位时期简单的看成是多个稳态状态下的连续过程. 假设 每一个稳态条件下 (即 $1 \mathrm{~d}$ 内) 磷释放率和沉降率保持不变, 水体体积不变, 单位时间流人水体流量 $=$ 流出水 体流量.

4) 因本研究主要目的为探寻磷在高水位期间的形态转化和循环机制, 所以不考虑水体中由于底栖动物 的扰动所导致的内源磷释放以及浮游动物和鱼类等对藻类滤食作用的影响.

5) 在高水位枯水季节, 因为水淹没土壤而释放的藻类生物量相对较少以及相关资料的缺乏, 藻类生物 量模型暂不考虑陆源输人的影响以及水位上升淹没消落带所带来的藻类生物量输人的影响.

6) 因在高水位期间该研究区域降雨量较少, 水淹没土壤而释放的正磷酸盐 (SRP) 相对较少以及相关资 料的缺乏,SRP 模型暂不考虑陆源输人的影响.

7) 在 SRP 模型中, 根据底泥和消落带土壤 TP 形态分布特征, 假设其活性形态部分释放的磷全部为 
$\mathrm{SRP}$, 即底泥和消落带释放的 $\mathrm{TP}=\mathrm{SRP}$.

根据以上假设, 藻类生物量概念性模型如下:

高阳平湖水中藻类生物量变化 $=$ 上游藻类生物量输人总量 + 高阳平湖陆源藻类生物量输人总量 $($ 本模 型假设为 0$)+$ 水位升高消落带所释放的藻类总量 (本模型假设为 0 ) - 高阳平湖下游藻类生物量输出总量 + 藻类生长所增加的生物量总量-藻类死亡所减少的生物量总量-藻类沉降所减少的生物量总量-浮游动物和 鱼类等滤食所消耗的藻类生物量总量 (本模型假设为 0 ).

SRP 概念性模型如下:

高阳平湖水中 SRP 总量的变化 $=$ 上游 $\mathrm{SRP}$ 输人总量 + 高阳平湖陆源 (点源、非点源) SRP 输人总量 $($ 本 模型假设为 0$)+$ 水位升高消落带 SRP 释放总量+底泥 SRP 释放总量-高阳平湖下游 SRP 输出总量-藻类通 过光合作用将 SRP 合成为有机磷 $(\mathrm{OP})$ 总量+细菌通过降解过程将 OP 分解成 SRP 总量.

\section{2 藻类生物量模型}

1) 输人项: 上游藻类生物量输人、高阳平湖陆源藻类生物量输人 (点、面源输人等, 本模型假设为 0 )、水 位升高消落带所释放的藻类生物量 ${ }^{[20-21]}$ (本模型假设为 0 ).

2) 输出项: 高阳平湖下游藻类生物量输出.

$3)$ 反应项: 藻类生长、藻类死亡 (呼吸、分解)、藻类沉降、浮游动物和鱼类等的滤食 (本公式不予考虑). 则公式为:

$$
V \cdot \frac{\mathrm{d} B i o M}{\mathrm{~d} t}=W_{\curlywedge}-Q \cdot B i o M+u \cdot B i o M \cdot V-M a \cdot B i o M \cdot V-v_{\text {Chl.a }} \cdot B i o M \cdot V
$$

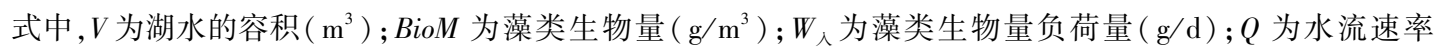
$\left(\mathrm{m}^{3} / \mathrm{d}\right) ; \mu$ 为藻类生长速率 $\left(\mathrm{d}^{-1}\right) ; M a$ 为藻类死亡率 $\left(\mathrm{d}^{-1}\right) ; v_{\text {Chl. }}$ 为藻类沉降速率 $\left(\mathrm{d}^{-1}\right)$.

对于藻类生物量模型而言, 各磷库的速率、通量计算公式见表 1 .

表 1 藻类生物量模型速率、通量计算公式

Tab.1 Formula of phosphorus fluxes and the rates of conversion of BioM model

\begin{tabular}{lcc}
\hline 磷库 & 速率计算公式 $/(\mathrm{g} / \mathrm{d})$ & 通量计算公式 $/\left(\mathrm{g} /\left(\mathrm{d} \cdot \mathrm{m}^{3}\right)\right)$ \\
\hline 藻类生物量净累积 & $W_{\text {人 }}-Q \cdot B i o M+u \cdot B i o M \cdot V-$ & $\left(W_{\text {人 }}-Q \cdot B i o M\right) / V+u \cdot B i o M-$ \\
藻类生物量上游输人 & $M a \cdot B i o M \cdot V-v_{\text {Chl.a }} \cdot B i o M \cdot V$ & $M a \cdot B i o M-v_{\text {Chl.a }} \cdot B i o M$ \\
藻类生物量下游输出 & $Q \cdot B i o M$ & $Q \cdot B i o M / V$ \\
藻类总增长 & $Q \cdot B i o M$ & $Q \cdot B i o M / V$ \\
藻类死亡 & $\mu \cdot B i o M \cdot V$ & $\mu \cdot B i o M$ \\
藻类沉降 & $M a \cdot B i o M \cdot V$ & $M a \cdot B i o M$ \\
\hline
\end{tabular}

\section{3 正磷酸盐 (SRP) 模型}

1) 输人项: 上游 SRP 输人、高阳平湖陆源 (点源、非点源) SRP 输人 (本模型中假设为 0 )、水位升高消落 带 SRP 释放、永久淹没区 SRP 释放.

2) 输出项 : 高阳平湖下游 SRP 输出.

3) 反应项: 水体内部磷形态发生的变化. 主要是两个方面,一是藻类通过光合作用将 SRP 合成为 OP, 对 SRP 而言, 这部分是水体中 SRP 的反应消耗; 二是细菌通过降解过程, 将 OP 分解成 SRP, 对 SRP 而言, 这部 分是水中 SRP 的反应产出. 则公式为:

$$
\begin{aligned}
& V \frac{\mathrm{d} P I}{\mathrm{~d} t}=W_{i}+v_{r 1} \cdot A_{\text {消落带 }} \cdot p s_{1}+v_{r 2} \cdot A_{\text {永久海没区 }} \cdot p s_{2}- \\
& Q \cdot P I-U P \cdot B i o M \cdot V+M a \cdot B i o M \cdot A P_{0} \cdot K_{m} \cdot \theta_{m}^{T-20} \cdot V
\end{aligned}
$$

式中, $V$ 为湖水的容积 $\left(\mathrm{m}^{3}\right) ; P I$ 为湖水正磷酸盐浓度 $\left(\mathrm{g} / \mathrm{m}^{3}\right) ; t$ 为时间 $(\mathrm{d}) ; W_{i}$ 为正磷酸盐负荷量 $(\mathrm{g} / \mathrm{d}) ; Q$

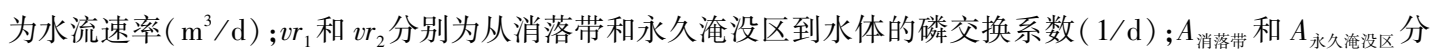


别为消落带和永久淹没区表面积 $\left(\mathrm{m}^{2}\right) ; p s_{1}$ 和 $p s_{2}$ 分别为消落带和永久淹没区土壤活性磷含量 $\left(\mathrm{g} / \mathrm{m}^{2}\right) ; U P$ 为 藻类对磷的吸收速率 $\left(\mathrm{d}^{-1}\right) ; A P_{0}$ 为藻中磷含量 $(\mathrm{g} / \mathrm{g}) ; K_{m}$ 为 $20^{\circ} \mathrm{C}$ 时 $\mathrm{OP}$ 的矿化率 $\left(\mathrm{d}^{-1}\right) ; \theta_{m}$ 为有机磷矿化的温 度系数.

对于 SRP 模型而言,各磷库的速率、通量计算公式见表 2.

表 2 SRP 模型速率和通量计算公式

Tab.2 Formula of phosphorus fluxes and the rates of conversion of SRP model

\begin{tabular}{|c|c|c|}
\hline 磷库 & 速率计算公式 & 通量计算公式 \\
\hline $\mathrm{SRP}$ 累积 & $\begin{array}{c}W_{i}+V_{r 1} \cdot A_{\text {消落带 }} \cdot p s_{1}+v_{r 2} \cdot A_{\text {永久淹没区 }} \cdot p s_{2}- \\
Q \cdot P I-U P \cdot B i o M \cdot V+M a \cdot B i o M \cdot \\
A P_{0} \cdot K_{m} \cdot \theta_{m}^{T-20} \cdot V\end{array}$ & $\begin{array}{c}\left(\begin{array}{c}W_{i}+V_{r 1} \cdot A_{\text {消落带 }} \cdot p s_{1}+ \\
v_{r 2} \cdot A_{\text {永久淹没区 }} \cdot p s_{2}-Q \cdot P I\end{array}\right) / V- \\
U P \cdot B i o M+M a \cdot B i o M \cdot \\
A P_{0} \cdot K_{m} \cdot \theta_{m}^{T-20}\end{array}$ \\
\hline $\mathrm{SRP}$ 上游输人 & $Q \cdot P I_{\text {入 }}$ & $Q \cdot P I_{\curlywedge} / V$ \\
\hline $\mathrm{SRP}$ 下游输出 & $Q \cdot P I$ & $Q \cdot P I / V$ \\
\hline 消耗 SRP 合成 OP & $U P \cdot B i o M \cdot V$ & $U P \cdot B i o M$ \\
\hline 藻类死亡释放 SRP & $M a \cdot B i o M \cdot A P_{0} \cdot K_{m} \cdot \theta_{m}^{T-20} \cdot V$ & $M a \cdot B i o M \cdot A P_{0} \cdot K_{m} \cdot \theta_{m}^{T-20}$ \\
\hline
\end{tabular}

初始条件下, 根据相关文献和前期研究结果, 对模型参数设置初始估值, 见表 3.

表 3 模型参数表

Tab.3 Parameters for model simulation

\begin{tabular}{|c|c|c|c|c|}
\hline 参数 & 符号 & 单位 & 取值 & 文献 \\
\hline $20^{\circ} \mathrm{C}$ 时消落带磷矿化率 & $K m_{2}$ & $d^{-1}$ & 0.05 & 实验测定 \\
\hline $20^{\circ} \mathrm{C}$ 时永久淹没区磷矿化率 & $\mathrm{Km}_{3}$ & $d^{-1}$ & 0.0025 & 文献[22-23] \\
\hline 消落带磷矿化温度系数 & $\theta m_{2}$ & - & 1.15 & 文献[23] \\
\hline 永久淹没区磷矿化温度系数 & $\theta m_{3}$ & - & 1.15 & 文献[23] \\
\hline 藻类沉降速率 & $v_{\text {Chl.a }}$ & $d^{-1}$ & 0.015 & 文献[24] \\
\hline 藻类最大生长速率 & $\mu_{\max }$ & $d^{-1}$ & 1.16 & 文献 $[25]$ \\
\hline 藻类生长最适温度 & $T_{\mathrm{opt}}$ & ${ }^{\circ} \mathrm{C}$ & 20 & 文献[26] \\
\hline $20^{\circ} \mathrm{C}$ 时温度经验常数 & $\theta_{\text {Chl.a }}^{\text {opt }}$ & - & 1.02 & 文献[27] \\
\hline 藻类生长最适光照强度 & $I_{S}$ & $\mu \mathrm{mol} /\left(\mathrm{m}^{2} \cdot \mathrm{s}\right)$ & 148.15 & 文献[26] \\
\hline 藻对磷的半饱和常数 & $K P$ & $\mathrm{~g} / \mathrm{m}^{3}$ & 0.18 & 文献 $[16,21]$ \\
\hline 藻类最大死亡率 & $M a_{\max }$ & $d^{-1}$ & 0.128 & 文献[21] \\
\hline 藻类死亡率的半饱和常数 & $K_{m 1}$ & $\mathrm{~g} / \mathrm{m}^{3}$ & 18 & 文献[21] \\
\hline 藻中磷含量 & $A P_{0}$ & $g / g$ & 0.00628 & 文献[16-17] \\
\hline $20^{\circ} \mathrm{C}$ 时有机磷的矿化率 & $K_{m}$ & $\mathrm{~d}^{-1}$ & 0.022 & 文献[22] \\
\hline 有机磷矿化的温度系数 & $\theta_{m}$ & - & 1.15 & 文献[22] \\
\hline 藻类的最大磷摄取率 & $U P_{\max }$ & $d^{-1}$ & 0.005 & 文献[16-17] \\
\hline 藻中最高磷浓度 & $F_{\max }$ & $g / g$ & 0.015 & 文献 $[16,22]$ \\
\hline 藻中最低磷浓度 & $F_{\min }$ & $g / g$ & 0.001 & 文献 $[16,22]$ \\
\hline
\end{tabular}

\section{4 模型实施与校准}

一般情况下总会有某些模拟值与实测值不完全拟合, 但两者变动趋势基本一致. 可以用校验期状态变 量校对误差 $Y$ 、平均状态变量校对误差 $R$ 和最大状态变量校对误差 $A$ 这 3 个校准指标来定量表示. 若 3 个指 标的值都比较小, 则可知此模型具有预测价值 ${ }^{[28-29]}$. 其公式分别为:

$$
\begin{gathered}
Y=\left\{\left[\sum\left(Y_{\mathrm{c}}-Y_{\mathrm{m}}\right)^{2}\right]^{1 / 2} / n\right\} / \bar{Y}_{\mathrm{m}} \times 100 \% \\
R=\left(\bar{Y}_{\mathrm{c}}-\bar{Y}_{\mathrm{m}}\right) / \bar{Y}_{\mathrm{m}} \times 100 \%
\end{gathered}
$$




$$
A=\left(Y_{\mathrm{c}, \text { max }}-Y_{\mathrm{m}, \text { max }}\right) / Y_{\mathrm{m}, \text { max }} \times 100 \%
$$

式中, $Y_{\mathrm{c}} 、 \bar{Y}_{\mathrm{c}}$ 和 $Y_{c, \text { max }}$ 分别为校验期模拟值、平均模拟值和最高模拟值, $Y_{\mathrm{m}} 、 \bar{Y}_{\mathrm{m}}$ 和 $Y_{\mathrm{m}, \text { max }}$ 分别为校验期实测值、 平均实测值和最高实测值.

表 4 研究期间高阳平湖磷-藻生态模型 2 个主要状态变量校准指标

Tab.4 Three main calibration indexes of state variables in Lake Gaoyang during the research

\begin{tabular}{lrrr}
\hline 状态变量 & $Y / \%$ & $R / \%$ & $A / \%$ \\
\hline $\mathrm{SRP}$ & 0.105 & 8.220 & -3.843 \\
藻类生物量 & 33.298 & -18.646 & -48.867 \\
\hline
\end{tabular}

由模型中 SRP 浓度和藻类生物量这 2 个主要状态变量的校验指标 $Y 、 R$ 和 $A$ 值可知 (表 4), SRP 浓度 和藻类生物量的 $R$ 绝对值都在 $20 \%$ 以内, 其中 SRP 浓度的 $Y 、 R$ 和 $A$ 的绝对值甚至在 $10 \%$ 以内, 而藻类生物 量的 $Y$ 值和 $A$ 值虽然相对较大, 但整体都在 50\% 以内, 在仅提供少量测量数据的情况下, 这样的差异是可以 接受的.

\section{3 结果与分析}

\section{1 高水位期间不同阶段各磷库磷的转化速率}

高阳平湖高水位时期不同磷库之间磷素的转化速率表明(图 3), SRP 累积速率和消落带磷交换速率在 整个高水位时期的变化趋势相同. 在蓄水期均呈现出先上升后下降的趋势, 蓄水期均值分别为 (34.94 $7.68) \times 10^{5}$ 和 $(29.13 \pm 4.99) \times 10^{5} \mathrm{~g} / \mathrm{d}$, 其中在 9 月 23 日均达到了整个高水位时期的最大值, 分别为 $48.33 \times 10^{5}$ $\mathrm{g} / \mathrm{d}$ 和 $40.57 \times 10^{5} \mathrm{~g} / \mathrm{d}$, 经过稳定期的起伏以后, 在水华期持续下降至最低值, 水华期的均值分别为 $(3.26 \pm$ $1.24) \times 10^{5}$ 和 $(5.53 \pm 1.03) \times 10^{5} \mathrm{~g} / \mathrm{d}$; 藻类生物量净累积速率和藻类总增长速率在整个高水位时期变化趋势 略有不同. 虽然在蓄水期均呈现出前期逐渐上升, 中、后期波动上升的状态, 并且在稳定期持续波动下降, 水 华期先下降后升高. 但藻类生物量净累积速率在蓄水期达到最大值 $\left(48.74 \times 10^{6} \mathrm{~g} / \mathrm{d}\right)$, 在水华期达到最小值 $\left(-35.83 \times 10^{6} \mathrm{~g} / \mathrm{d}\right)$. 而藻类总增长速率在水华期变化巨大, 先下降至整个高水位时期的最低值 $\left(31.90 \times 10^{5}\right.$ $\mathrm{g} / \mathrm{d}$ ), 后又陡升至最高值 $\left(937.37 \times 10^{5} \mathrm{~g} / \mathrm{d}\right)$, 水华期均值为 $(193.26 \pm 221.06) \times 10^{5} \mathrm{~g} / \mathrm{d}$; 藻类沉降速率和藻类 死亡速率在高水位时期的蓄水期和稳定期变化不同, 藻类沉降速率在蓄水期前期小幅上升, 在蓄水期中后 期开始下降直到稳定期结束. 藻类死亡速率在蓄水期和稳定期均保持在较低水平, 略有浮动. 但在水华期, 藻类沉降速率和藻类死亡速率均呈现上升趋势, 并达到了整个高水位时期的最大值, 分别为 $7.36 \times 10^{5}$ 和 $62.83 \times 10^{6} \mathrm{~g} / \mathrm{d}$. 水华期均值分别为 $(47.03 \pm 30.02) \times 10^{5}$ 和 $(199.52 \pm 200.93) \times 10^{5} \mathrm{~g} / \mathrm{d}$; 藻类消耗 SRP 速率和藻 类死亡释放 SRP 速率的变化趋势在整个高水位时期表现基本相同. 藻类消耗 SRP 速率先上升,在蓄水期前 期便开始下降一直到稳定期, 保持在最低水平, 水华期持续上升至最高值. 3 个时期的均值分别为 $(8.83 \pm$ $2.38) \times 10^{4} 、(9.51 \pm 2.61) \times 10^{4} 、(7.51 \pm 8.44) \times 10^{4} \mathrm{~g} / \mathrm{d}$. 藻类死亡释放 SRP 速率先上升至蓄水期末期开始下降 一直到水华期中期至最低值, 然后开始上升至整个高水位期间的最大值. 3 个时期的均值分别为 $(569.14 \pm$ $158.56) 、(92.74 \pm 52.62) 、(872.41 \pm 903.72) \mathrm{g} / \mathrm{d}$. 永久淹没区磷交换速率在整个高水位时期一直呈下降趋 势, 3 个时期的磷交换速率均值分别为 $(68.32 \pm 19.91) \times 10^{4} 、(24.99 \pm 5.46) \times 10^{4} 、(9.59 \pm 2.94) \times 10^{4} \mathrm{~g} / \mathrm{d}$.

\section{2 高水位期间不同阶段各磷库磷通量}

SRP 累积通量与消落带、永久淹没区水土界面磷交换通量具有相同的变化趋势 ( 图 4). 在蓄水期达到 最大值, 分别为 $(27.525 \pm 4.128) \times 10^{-3} 、(22.230 \pm 5.692) \times 10^{-3}$ 和 $(5.653 \pm 3.316) \times 10^{-3} \mathrm{~g} /\left(\mathrm{d} \cdot \mathrm{m}^{3}\right)$. 稳定期随着 时间增加而逐渐降低, 在水华期达到最低值, 分别为 $(0.576 \pm 0.086) \times 10^{-3} 、(3.969 \pm 0.436) \times 10^{-3}$ 和 $(0.683 \pm$ $0.173) \times 10^{-3} \mathrm{~g} /\left(\mathrm{d} \cdot \mathrm{m}^{3}\right)$. 即在蓄水期出现磷的累积, 而在高水位中期开始随即出现累积磷量下降; 藻磷净累 积通量在稳定期取得最大值, 为 $(0.526 \pm 0.079) \times 10^{-3} \mathrm{~g} /\left(\mathrm{d} \cdot \mathrm{m}^{3}\right)$, 呈现先增加后减少的趋势; 藻类死亡通量、 藻类死亡形成碎屑磷通量、藻磷沉降通量、㩰类消耗 SRP 通量以及藻类死亡释放 SRP 通量变化趋势大体相 同, 在稳定期达到最低, 都呈先降低后增加的变化趋势, 其中藻类消耗 SRP 通量在蓄水期取得最大值, 为 

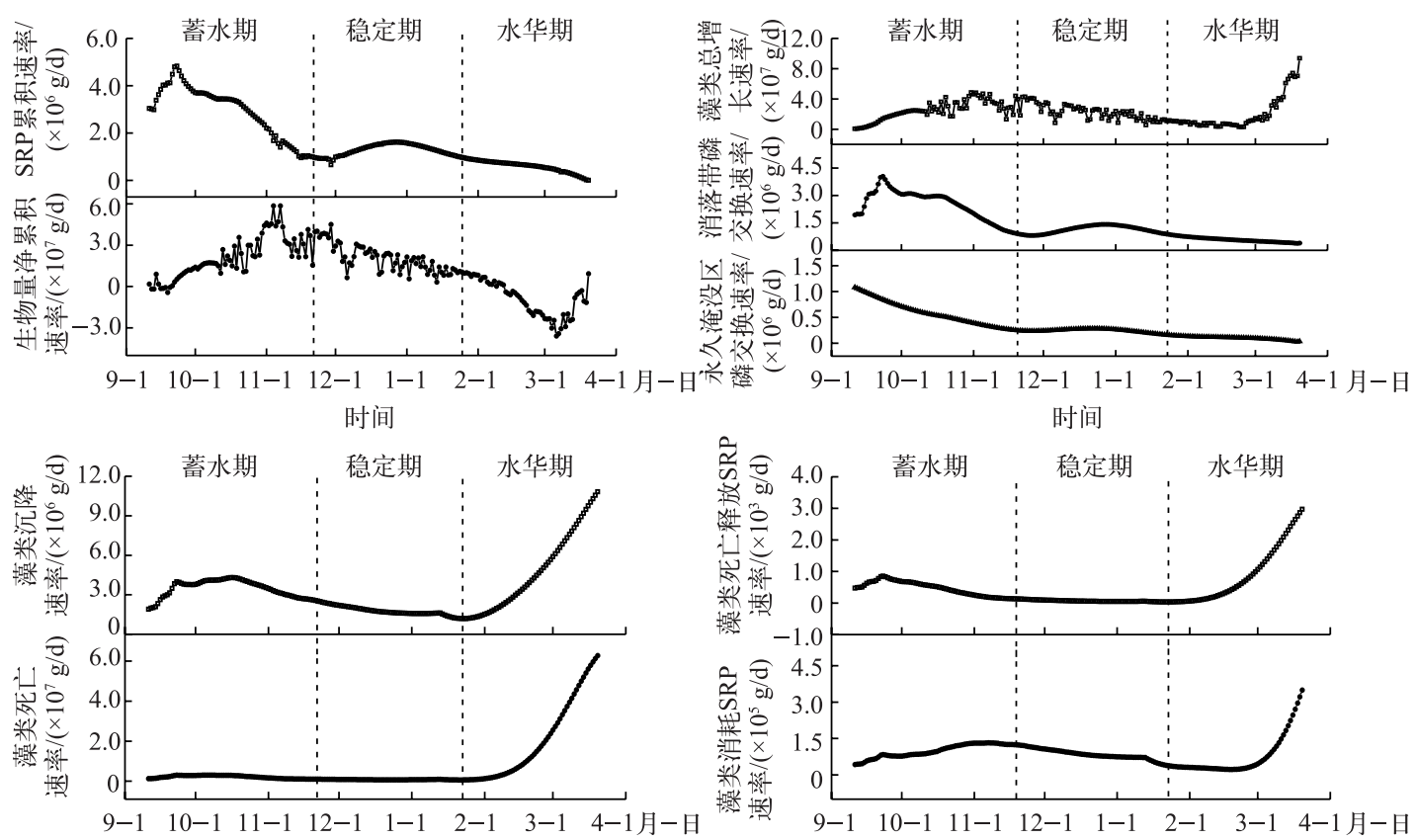

图 3 研究期间高阳平湖高水位时期不同磷库之间磷素的转化速率

Fig.3 Rates of conversion between the different phosphorus pools in Lake Gaoyang during high water level stage

$(0.637 \pm 0.096) \times 10^{-3} \mathrm{~g} /\left(\mathrm{d} \cdot \mathrm{m}^{3}\right)$, 而藻类死亡形成碎屑磷通量、藻磷沉降通量和藻类死亡释放 SRP 通量都在 水华期达到最大值, 分别为 $(0.969 \pm 0.145) \times 10^{-3} 、(0.222 \pm 0.031) \times 10^{-3}$ 和 $(6.758 \pm 0.676) \times 10^{-6} \mathrm{~g} /\left(\mathrm{d} \cdot \mathrm{m}^{3}\right)$.

\section{4 讨论}

\section{1 高阳平湖高水位时期磷素转化途径特征分析}

蓄水期, 前期不断蓄水以及水位上升导致淹没消落带面积增多, 因而在蓄水初期 SRP 累积速率、消落带 水土界面磷交换速率不断增加, 消落带水土界面磷交换速率在蓄水期达到了最大值. 整个高水位时期, 消落 带水土界面磷交换速率比永久淹没区水土界面磷交换速率要高, 淹没消落带磷释放可能是高阳平湖 SRP 累 积的主要来源. 大量外源性藻类生物量的输人也使得此时藻类总增长速率增加 ${ }^{[30-31]}$, 从而导致水中以藻类 生物量为本底值的藻类生物量净累积速率、㩰类死亡速率、藻类沉降速率、藻类消耗 SRP 速率和藻类死亡释 放 SRP 速率随着藻类生物量的增加而增加. 由于蓄水水位升高和顶托而导致的大量磷积累, 可能存在藻对 磷的 “奢侈性” 吸收 ${ }^{[32]}$. 由前期研究可知, 此时湖水中藻类生长更可能受光热条件限制, 外源性输人藻类和 本地藻类随着外界光热条件的变化而呈波动性增长, 随着藻类消耗 SRP 速率的持续增加, 加之消落带和永 久淹没区在蓄水初期具有较大磷释放速率而到了蓄水中后期磷释放速率逐渐减缓 ${ }^{[6,33-35]}$, 磷释放量逐渐达 到最大值,动力学趋向于平衡,因此在蓄水中后期 SRP 累积速率开始下降.

稳定期, 在经历蓄水期的浸泡后淹没消落带和永久淹没区土壤磷释放速率在此时已达到动态平衡, 因 而消落带和永久淹没区水土界面磷交换速率变化较为平缓, SRP 累积速率在此时变幅也比较小, 而且都表 现为正值. 在稳定期, 对藻类生长构成限制的主要为水温、太阳辐射强度和水动力条件的下降 ${ }^{[15,36-37]}$. 高水 位稳定期是藻类的非生长季节, 因而藻类总增长速率和藻类生物量净累积速率出现平缓下降趋势. 如何在 低能量条件下维持自身生长成为此时藻类的主要生存机制. 因此, 藻类死亡速率、藻类消耗 SRP 速率、藻类 死亡释放 SRP 速率和藻类沉降速率也逐渐降低并稳定在较低状态.

水华期, 前期受出湖水量增加和稳定期过渡的影响, 藻类总增长速率和生物量净累积速率与稳定期趋 势保持一致,呈下降趋势. 在水华期中后期, 气温逐步回暖, 水位逐渐下降, 水流速度有所增加, 光热条件和 

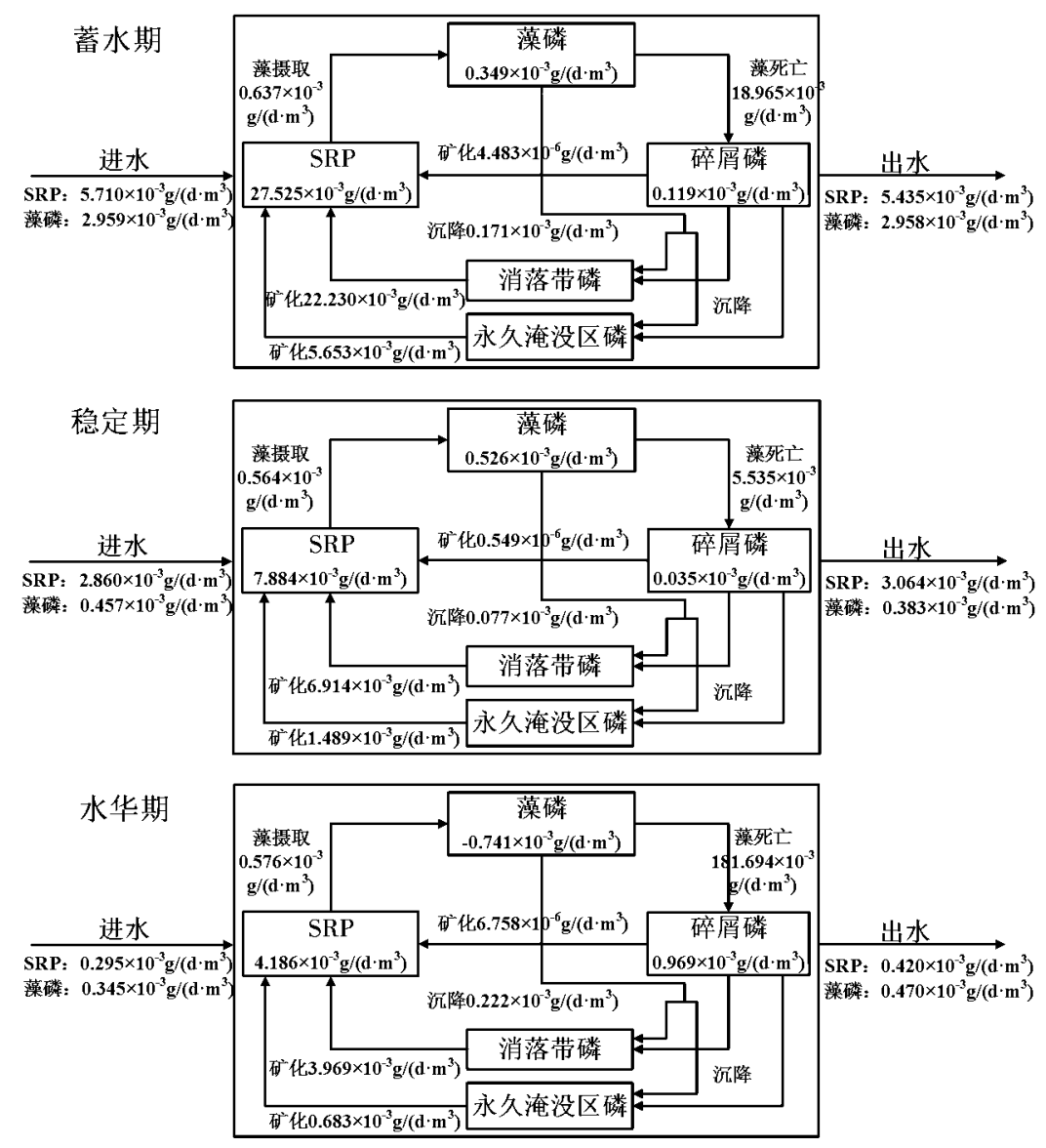

图 4 研究期间高阳平湖高水位期间不同阶段各磷库磷通量

Fig.4 Phosphorus fluxes of the different phosphorus pools in Lake Gaoyang during the research

水动力条件逐渐适宜藻类的生长, 藻类总增长速率和生物量净累积速率在此时出现陡增. 藻类的大量生长 和高阳平湖泄水带来的磷流失的双重作用下, 必然使得藻类消耗 SRP 速率出现陡增, SRP 累积速率逐渐下 降,一直降至研究期间的最低值. 此时的外界磷浓度较低, 藻对磷的吸收存在两种主动方式: 一是诱导产生 碱性磷酸酶( APA ) 分解前期累积在水中的有机磷, 释放出能被其直接利用的 $\mathrm{SRP}^{[38]}$; 二是利用前期累积储 存在体中的磷或脂肪等来提供生长所需的磷源,这两种方式的直接磷源皆来自于前期所累积的磷.

在高水位水华期, 藻磷和水磷是一个快速转换的过程, 相应的随着藻类生物量的增长以及微生物活性 的提高, 藻类死亡速率、死亡释放 SRP 速率和藻类沉降速率也逐渐增强.

\section{2 高阳平湖高水位时期各磷库磷交换通量估算}

在前期研究的基础上, 通过建立高水位时期高阳平湖磷-藻类生态模型对高阳平湖累积总磷的转化途 径和通量进行模拟 (图 5). 从正磷酸盐所代表的无机磷来看, 高水位时期高阳平湖 SRP 主要通过上游输人、 永久淹没区和淹没消落带释放和藻类等有机磷死亡释放这 4 种途径来收人,通过下游和被藻类消耗合成有 机磷这 2 种途径来支出. 对于 SRP 收人途径来说, 淹没消落带磷释放是冬季高水位时期高阳平湖 SRP 的主 要来源, 整个高水位时期输人 $20.009 \times 10^{-3} \mathrm{~g} /\left(\mathrm{d} \cdot \mathrm{m}^{3}\right)$, 占收人总通量的 $72.69 \%$, 永久淹没区磷释放是次要 输人来源, 整个高水位时期输人 $4.673 \times 10^{-3} \mathrm{~g} /\left(\mathrm{d} \cdot \mathrm{m}^{3}\right)$, 占收人总通量的 $16.98 \%$; 对于 SRP 支出途径来说, 下游输出是 SRP 支出的主要途径,支出总量为 $2.895 \times 10^{-3} \mathrm{~g} /\left(\mathrm{d} \cdot \mathrm{m}^{3}\right)$, 占支出总通量的 $83.14 \%$.

从藻类生物量所代表的有机磷来看, 高水位时期高阳平湖藻磷主要通过上游藻磷输人和藻类自身生长 


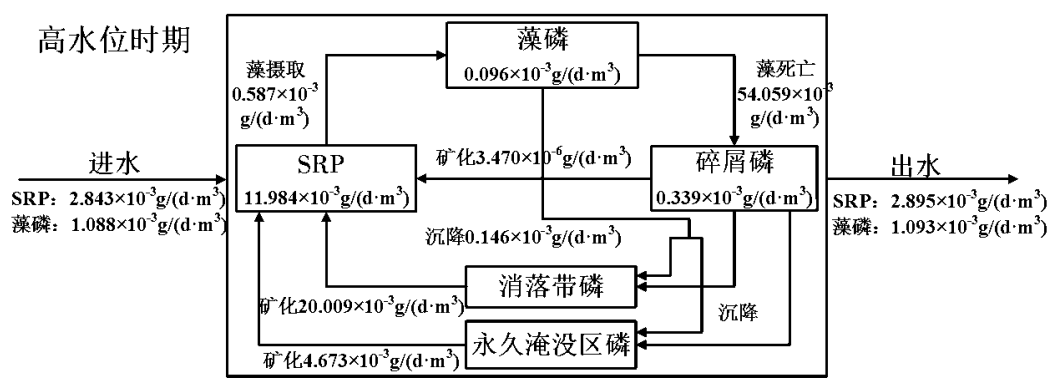

图 5 研究期间高阳平湖高水位时期累积磷的转化通量示意

Fig.5 Conversion of fluxes from TP accumulation in Lake Gaoyang during high water level stage

这 2 种途径来收人, 通过下游藻类磷输出、藻类死亡形成碎屑磷和藻磷沉降这 3 种途径来支出. 对于藻磷收 人途径来说, 上游藻类生物量输人成为藻类生物量收人的首要来源, 输人总量为 $1.088 \times 10^{-3} \mathrm{~g} /\left(\mathrm{d} \cdot \mathrm{m}^{3}\right)$, 占 总收人通量的 $64.96 \%$; 对于藻磷支出途径来说, 下游输出是藻类生物量支出的首要途径, 输出总量为 $1.093 \times$ $10^{-3} \mathrm{~g} /\left(\mathrm{d} \cdot \mathrm{m}^{3}\right)$, 占支出总通量的 69.26\%. 其次是藻类死亡, 死亡总通量为 $54.059 \times 10^{-3} \mathrm{~g} /\left(\mathrm{d} \cdot \mathrm{m}^{3}\right)$, 占支出 总通量的 $21.49 \%$, 而藻类沉降只占支出总通量的 $9.25 \%$, 沉降总量为 $0.146 \times 10^{-3} \mathrm{~g} /\left(\mathrm{d} \cdot \mathrm{m}^{3}\right)$. 可以看出, 冬 季高水位时期上游来水和下游出水所造成的外源性 SRP 和藻磷输移量基本相当, 处于一种动态平衡状态, 由此可以推测藻类在高水位末期发生水华更大可能是受内源性因素的影响. 淹没消落带磷释放是高阳平湖 SRP 累积的主要来源, 其释放量与被淹没消落带的面积和消落带水土界面磷交换速率有关, 淹没面积越多, 磷交换速率越高, 磷释放量越多,对整个磷循环的贡献越大.

藻类对冬季高水位期间所积累磷的利用率较低. 水华期, 由于光热条件和水动力条件逐渐适宜藻类生 长, 藻磷合成效率 (藻类在光合作用中用于合成 OP 的 SRP 的量占总磷累积量的百分比) 达到最大 $(14.4 \%)$. 稳定期和蓄水期的藻磷合成效率分别为 $7.05 \%$ 和 $2.275 \%$, 冬季高水位时期高阳平湖 SRP 的主要来源为淹没 消落带磷释放 (占总输人通量的 $72.69 \%$ ), 可知冬季藻类水华所需的 SRP 主要来自于高水位蓄水淹没大量 消落带, 但冬季藻类水华过程中对 SRP 的转化利用能力较低.

\section{5 结论}

高阳平湖高水位时期的蓄水期、稳定期和水华期这 3 个阶段中各磷库磷的转化速率和通量各不相同, 磷的形态转化和循环机制贯穿于整个高水位时期. 蓄水期, 消落带水土界面磷交换速率和磷释放通量在蓄 水期达到了整个高水位时期的最大值, 藻类中的 SRP 累积速率达到最大, SRP 得到显著积累. 稳定期, 水柱 中营养物质相对丰足, 为藻类的非生长季节, 如何在低能量条件下维持自身生长成为此时藻类的主要生存 机制. 水华期, 光热条件和水动力条件逐渐适宜澡类生长, 藻类通过光合作用分解在蓄水期积累的 SRP 合成 $\mathrm{OP}$ 实现增殖,形成冬季末水华, 当外界磷浓度较低时, 藻类、细菌等产生碱性磷酸酶分解水中的有机磷或者 前期累积储存在体中的磷或脂肪等来补充生长所需的 $\mathrm{SRP}^{[38]}$, 藻磷和水磷是一个快速转换的过程, 其磷源 均来自于前期所累积的总磷.

淹没消落带磷释放是冬季高水位时期高阳平湖 SRP 的主要来源 (占总输人通量的 $72.69 \%$ ), 冬季藻类 水华所消耗的 SRP 主要来自于高水位蓄水时淹没的大量消落带, 其释放量与被淹没消落带的面积和消落带 水土界面磷交换速率有关, 淹没面积越多, 磷交换速率越高, 磷释放量越多, 对整个磷循环的贡献越大. 水华 期间藻磷的合成效率最大仅为 $14.4 \%$, 藻类对冬季高水位期间所积累 SRP 的利用量依然相对较低.

\section{6 参考文献}

[ 1 ] Guo Jinsong, Zhang Chao, Fang Fang et al. Study on features of phytoplankton community in Xiaojiang River Backwater Area in Three Gorges Reservoir during spring and summer time. Science \& Technology Review, 2008, 26(17) : 70-75(in Chinese with English abstract). [ 郭劲松, 张超, 方芳等. 三峡水库小江回水区水华高发期浮游植物群落结构特征 
研究. 科技导报, 2008, 26(17): 70-75. ]

[ 2 ] Li Zhe, Guo Jinsong, Fang Fang et al. Seasonal variation of cyanobacteria and its potential relationship with key environmental factors in Xiaojiang Backwater area, Three Gorges Reservoir. Chinese Journal of Environmental Science, 2010, 31 (2) : 301-309 (in Chinese with English abstract). [李哲, 郭劲松, 方芳等. 三峡小江回水区蓝藻季节变化及其与主 要环境因素的相互关系. 环境科学, 2010, 31(2) : 301-309.]

[ 3 ] Guo Jinsong, Li Zhe, Zhang Cheng et al. Canonical correspondence analysis of phytoplankton assemblages and environmental factors in Xiaojiang Backwater Area, Three Gorges Reservoir. Journal of Yangtze River Scientific Research Institute, 2010, 27 (10): 60-64, 87 (in Chinese with English abstract). [ 郭劲松, 李哲, 张呈等. 三峡小江回水区藻类集群与 主要环境要素的典范对应分析研究. 长江科学院院报, 2010, 27(10): 60-64, 87.]

[ 4 ] Yuan Hui, Wang Li'ao, Hu Gang et al. Release of N, P from submerged soil in the shore-area of Three Gorges Reservoir. Research of Environmental Sciences, 2008, 21(1): 103-106(in Chinese with English abstract). [袁辉, 王里奥, 胡刚 等. 三峡库区消落带受淹土壤氮和磷释放的模拟实验. 环境科学研究, 2008, 21(1): 103-106.]

[ 5 ] Yuan Hui, Huang Chuan, Cui Zhiqiang et al. Forecast of the relation between the water quality and the wet-dry zone of the three Gorges area. Journal of Chongqing University (Natural Science Edition), 2007, 30(9): 134-138(in Chinese with English abstract). [袁辉, 黄川, 崔志强等. 三峡库区消落带与水环境响应关系预测. 重庆大学学报: 自然科学版, $2007,30(9):$ 134-138.]

[ 6 ] Wang Li'ao, Huang Chuan, Zhan Yanhui et al. Flooding and subsequent air-drying on adsorption, desorption and release of phosphorus of soil in drawdown areas in Three Gorges Reservoir. Resources and Environment in the Yangtze Basin, 2006, 15(5): 593-597 (in Chinese with English abstract). [王里奥, 黄川, 詹艳慧等. 三峡库区消落带淹水一落干过程土 壤磷吸附一解吸及释放研究. 长江流域资源与环境, 2006, 15(5): 593-597.]

[ 7 ] Li Zhe, Fang Fang, Guo Jinsong et al. Spring algal bloom and nutrients characteristics in Xiaojiang River backwater area, Three Gorge Reservoir, 2007. J Lake Sci, 2009, 21 ( 1) : 36-44( in Chinese with English abstract). DOI: 10.18307/2009. 0105. [李哲, 方芳, 郭劲松等. 三峡小江回水区段 2007 年春季水华与营养盐特征. 湖泊科学, 2009, 21 (1)： 36-44.]

[ 8 ] Li Zhe, Wang Sheng, Guo Jinsong et al. Phytoplankton diversity in backwater area of the Pengxi River in the Three Gorges Reservoir before and after $156 \mathrm{~m}$ impoundment. J Lake Sci, 2012, 24(2) : 227-231 (in Chinese with English abstract). DOI: 10.18307/2012.0209. [李哲, 王胜, 郭劲松等. 三峡水库 $156 \mathrm{~m}$ 蓄水前后澎溪河回水区藻类多样性变化特征. 湖泊科学, 2012, 24(2): 227-231.]

[ 9 ] Fang Fang, Li Zhe, Tian Guang et al. Seasonal variation of phosphorus in Xiaojiang Backwater Area, Three Gorges Reservoir. Environmental Science, 2009, 30(12) : 3488-3493( in Chinese with English abstract). [方芳, 李哲, 田光等. 三峡 小江回水区磷素赋存形态季节变化特征及其来源分析. 环境科学, 2009, 30(12): 3488-3493.]

[10] He Bin, Li Zhe, Feng Jing. Total phosphorus model in Lake Gaoyang during the period of high water level in Pengxi River backwater zone, Three Gorges Reservoir. J Lake Sci, 2016, 28(2) : 295-302 (in Chinese with English abstract). DOI: 10.18307/2016.0208. [赫斌, 李哲, 冯婧等. 三峡澎溪河回水区高水位期间高阳平湖总磷模型. 湖泊科学, 2016, 28(2) : 295-302.]

[11] Huang Xuanmin. The research of distribution and exchange flux of soil carbon, nitrogen and phosphorus in water-level-fluctuating zone, Three Gorges Reservoir[Dissertation]. Chongqing: Chongqing University, 2012(in Chinese with English abstract). [ 黄轩民. 三峡水库消落带土壤碳氮磷分布及其交换通量研究 [ 学位论文]. 重庆: 重庆大学, 2012.]

[12] Zhang Bin. Study on distribution characteristics and flux of organic matter, nitrogen and phosphorus in the soil of WLFZ of Three Gorges Reservoir[Dissertation]. Chongqing: Chongqing University, 2013(in Chinese with English abstract). [张 涁. 三峡水库消落带土壤有机质、氮、磷分布特征及通量研究 [学位论文]. 重庆: 重庆大学, 2013.]

[13] Ministry of Environmental Protection of the People's Republic of China, Editorial Board of Water and Wastewater Monitoring and Analysis Methods ed. Water and wastewater monitoring and analysis methods: 4th edition. Beijing: China Environmental Science Press, 2002: 243-285(in Chinese). [国家环境保护总局《水和废水监测分析方法编委会》. 水和 废水监测分析方法: 第 4 版. 北京: 中国环境科学出版社, 2002: 243-285.]

[14] Lu Rukun ed. Soil agricultural chemical analysis method. Beijing: China Agricultural Science and Technolog Press, 2000 (in Chinese). [鲁如坤. 土壤农业化学分析方法. 北京: 中国农业科技出版社, 2000.]

[15] Li Zhe. On the habitats transition and succession traits of phytoplankton assemblages in the backwater area of Xiaojiang 
River during preliminary operation stage of the Three Gorges Reservoir[Dissertation]. Chongqing: Chongqing University, 2009 (in Chinese with English abstract). [李哲. 三峡水库运行初期小江回水区藻类生境变化与群落演替特征研究 [学位论文]. 重庆: 重庆大学, 2009.]

[16] Xu Qiujin, Qin Boqiang, Chen Weimin et al. Ecological simulation of algae growth in Taihu Lake. J Lake Sci, 2001,13 (2) : 149-157 (in Chinese with English abstract). DOI : 10.18307/2001.0208. [许秋瑾, 秦伯强, 陈伟民等. 太湖藻类 生长模型研究. 湖泊科学, 2001, 13(2): 149-157.]

[17] Quan Weimin. Assessment and model application of water body eutrophication in Qiandaohu Lake[Dissertation]. Hangzhou: Zhejiang University, 2002 (in Chinese with English abstract). [ 全为民. 千岛湖富营养化评价及其模型应用研究 [学位论文]. 杭州: 浙江大学, 2002.]

[18 ] Li Z, Zhang Z, Xiao Y et al. Spatio-temporal variations of carbon dioxide and its gross emission regulated by artificial operation in a typical hydropower reservoir in China. Environmental Monitoring and Assessment, 2014, 186( 5) : 3023-3039.

[19] Jiang Tao, Guo Jingsong, Li Zhe et al. Air-water surface greenhouse gas flux in Pengxi River at different operational stages of the Three Gorges Reservoir, Chinese. Journal of Environmental Science, 2012, (5) : 1463-1470(in Chinese with English abstract). [蒋滔, 郭劲松, 李哲等. 三峡水库不同运行状态下支流澎溪河水-气界面温室气体通量特征初探. 环境科学, 2012, (5): 1463-1470.]

[20] Liu Yongding, Li Shanghao. On Soil algae and their physiological ecology. Acta Hydrobiologica Sinica, 1993, 17(3): 272-277 (in Chinese with English abstract). [刘永定, 黎尚豪. 土壤藻类及其生理生态. 水生生物学报, 1993,17 (3) : 272-277.]

[21] Long TY, Wu L, Meng GH et al. Numerical simulation for impacts of hydrodynamic conditions on algae growth in Chongqing Section of Jialing River, China. Ecological Modelling, 2011, 222(1) : 112-119.

[22] Ruan Jingrong, Cai Qinghua, Liu Jiankang. East Lake phosphorus-phytoplankton dynamics model, Wuhan. Acta Hydrobiologica Sinica, 1988, 12(4) : 289-307 (in Chinese with English abstract). [阮景荣, 蔡庆华, 刘建康. 武汉东湖的磷浮游植物动态模型. 水生生物学报, 1988, 12(4): 289-307.]

[23] Pei Hongping, Wang Weiwei, He Jingtu et al. A phosphorus circulation dynamics model in the ecosystem for the west lake after drawing, Hangzhou. Acta Ecologica Sinica, 1998, 18(6) : 648-653 (in Chinese with English abstract). [裴洪平, 王 维维,何金土. 杭州西湖引水后生态系统中磷循环模型. 生态学报, 1998, 18(6): 648-653.]

[24] Xu Yunlin, Li Lixin, Li Li. The research of lake algae growth kinetics in Jingpohu. Acta Scientiarum Naturalium Universitatis Pekinensis, 1991, 27(6) : 725-737 (in Chinese with English abstract). [徐云麟, 李立新, 李莉. 湖泊藻类生长动 力学研究——以镜泊湖为例. 北京大学学报 (自然科学版), 1991, 27(6): 725-737.]

[25] Zhou Xianjie. Experimental study on eutrophication model statistics and algae growth of secondary rivers in the Three Gorges Reservoir Area[Dissertation]. Chongqing: Chongqing University, 2008(in Chinese with English abstract). [ 周贤杰. 三峡库区次级河流富营养化模型统计与藻类生长的试验研究 [学位论文]. 重庆: 重庆大学, 2008.]

[26] Zhou Xianjie, Luo Guyuan, Yang Qingling. Effects of different environmental factors on algal growth in the backwater reaches of the tributary to the Three-Gorges Reservoir: A simulation. Acta Scientiae Circumstantiae, 2008, 28(3) : 558-562 (in Chinese with English abstract). [周贤杰, 罗固源, 杨清玲等. 三峡库区次级河流回水区环境因子对藻类生长影 响的模拟实验研究. 环境科学学报, $2008,28(3)$ : 558-562.]

[27] Mao J, Chen Q, Chen Y. Three-dimensional eutrophication model and application to Taihu Lake, China. Journal of Environmental Sciences, 2008, 20(3): 278-284.

[28] Jørgensen SE, Bendoricchio G. Fundamentals of ecological modelling. Access Online via Elsevier, 2001.

[29] Pei H, Ma J. Study on the algal dynamic model for West Lake, Hangzhou. Ecological Modelling, 2002, 148(1) : 67-77.

[30] Li Zhe, Zhang Zengyu, Yang Zhonghua et al. Effects of flow speed on the change of in situ growth rates of algae in Pengxi River backwater zone, Three Gorges Reservoir. J Lake Sci, 2015, 27(5) : 880-886( in Chinese with English abstract). DOI : 10.18307/2015.0514. [李哲, 张曾宇, 杨中华等. 三峡澎溪河回水区流速对藻类原位生长速率的影响. 湖泊 科学, $2015,27(5): 880-886$. ]

[31] Feng Jing, Li Zhe, Yan Bin et al. In situ growth rate of dominant algae species in Pengxi River of the Three Gorges Reservoir. J Lake Sci, 2014, 26(2) : 235-242 (in Chinese with English abstract). DOI: 10.18307/2014.0210. [冯婧, 李哲, 间斌等. 三峡水库不同运行阶段澎溪河典型优势藻原位生长速率. 湖泊科学, 2014, 26(2) : 235-242.]

[32] Jr Bierman V, Dolan D. Modeling of phytoplankton-nutrient dynamics in Saginaw Bay, Lake Huron. Journal of Great Lakes 
Research, 1981, 7(4) : 409-439.

[33] Jin Danyue, Wang Shengrui, Bu Qingyun. Phosphate release kinetics characteristics of the sediments from shallow lakes in the middle and lower reaches of the Yangtze Rive. Ecology and Environment, 2007, 16(3) : 725-729(in Chinese with English abstract). [金丹越, 王圣瑞, 步青云. 长江中下游浅水湖泊沉积物磷释放动力学. 生态环境, 2007,16 (3) : 725-729.]

[34] Kim LH, Choi E, Stenstrom MK. Sediment characteristics, phosphorus types and phosphorus release rates between river and lake sediments. Chemosphere, 2003, 50(1): 53-61.

[35] McDowell R, Sharpley A. Phosphorus solubility and release kinetics as a function of soil test P concentration. Geoderma, 2003, 112(1): 143-154

[36] Wang Xiaoqing. The Three Gorges Reservoir area of Pengxi River eutrophication hydrodynamic and water quality coupling model research[Dissertation]. Chongqing: Chongqing University, 2012(in Chinese with English abstract). [王晓青. 三 峡库区澎溪河 (小江) 富营养化及水动力水质耦合模型研究 [学位论文]. 重庆: 重庆大学, 2012.]

[37] Liao Ping'an, Hu Xiulin. Experimental study on the effect of flow velocity on algal growth. Beijing Water Resources, 2005, (2) : 12-14 (in Chinese with English abstract). [廖平安, 胡秀琳. 流速对藻类生长影响的试验研究. 北京水利, 2005, (2): 12-14.]

[38 ] Zhang Ping, Feng Jing, Li Zhe et al. Alkaline phosphatase activity and its kinetics in Lake Gaoyang, Pengxi River during high water level of the Three Gorges Reservoir. J Lake Sci, 2015, 27(4) : 629-636(in Chinese with English abstract). DOI: $10.18307 / 2015.0410$. [张萍, 冯婧, 李哲等. 三峡澎溪河高阳平湖高水位时碱性磷酸酶活性及其动力学特 征. 湖泊科学, 2015, 27(4): 629-636. 